\title{
Multi-System Deconditioning in 3-Day Dry Immersion without Daily Raise
}

\author{
Steven De Abreu ${ }^{1 \dagger}$, Liubov Amirova ${ }^{1,2 \dagger}$, Ronan Murphy ${ }^{3}$, Robert Wallace ${ }^{3}$, \\ Laura Twomey ${ }^{3}$, Guillemette Gauquelin-Koch ${ }^{4}$, Veronique Raverot ${ }^{5}$, Françoise Larcher ${ }^{6}$, \\ Marc-Antoine Custaud ${ }^{1,7 *}$ and Nastassia Navasiolava ${ }^{7}$ \\ ${ }^{1}$ Mitovasc, UMR Institut National de la Santé et de la Recherche Médicale 1083, Centre National de la Recherche \\ Scientifique 6015, Université d'Angers, Angers, France, ${ }^{2}$ Russian Federation State Research Center, Institute of Biomedical \\ Problems, Russian Academy of Sciences, Moscow, Russia, ${ }^{3}$ Center for Preventive Medicine, School of Health and Human \\ Performance, Dublin City University, Dublin, Ireland, ${ }^{4}$ Centre National d'Etudes Spatiales, Paris, France, ${ }^{5}$ Hospices Civils de \\ Lyon, Lyon, France, ${ }^{6}$ Laboratoire de Biochimie, Centre Hospitalier Universitaire d'Angers, Angers, France, ${ }^{7}$ Centre de \\ Recherche Clinique, Centre Hospitalier Universitaire d'Angers, Angers, France
}

Dry immersion (DI) is a Russian-developed, ground-based model to study the physiological effects of microgravity. It accurately reproduces environmental conditions of weightlessness, such as enhanced physical inactivity, suppression of hydrostatic pressure and supportlessness. We aimed to study the integrative physiological responses to a 3-day strict DI protocol in 12 healthy men, and to assess the extent of multi-system deconditioning. We recorded general clinical data, biological data and evaluated body fluid changes. Cardiovascular deconditioning was evaluated using orthostatic tolerance tests (Lower Body Negative Pressure + tilt and progressive tilt). Metabolic state was tested with oral glucose tolerance test. Muscular deconditioning was assessed via muscle tone measurement.

Results: Orthostatic tolerance time dropped from $27 \pm 1$ to $9 \pm 2$ min after DI. Significant impairment in glucose tolerance was observed. Net insulin response increased by $72 \pm 23 \%$ on the third day of DI compared to baseline. Global leg muscle tone was approximately $10 \%$ reduced under immersion. Day-night changes in temperature, heart rate and blood pressure were preserved on the third day of DI. Day-night variations of urinary $\mathrm{K}^{+}$diminished, beginning at the second day of immersion, while 24-h $\mathrm{K}^{+}$excretion remained stable throughout. Urinary cortisol and melatonin metabolite increased with $\mathrm{DI}$, although within normal limits. A positive correlation was observed between lumbar pain intensity, estimated on the second day of DI, and mean 24-h urinary cortisol under DI. In conclusion, DI represents an accurate and rapid model of gravitational deconditioning. The extent of glucose tolerance impairment may be linked to constant enhanced muscle inactivity. Muscle tone reduction may reflect the reaction of postural muscles to withdrawal of support. Relatively modest increases in cortisol suggest that DI induces a moderate stress effect. In prospect, this advanced ground-based model is extremely suited to test countermeasures for microgravity-induced deconditioning and physical inactivity-related pathologies.

Keywords: modeled weightlessness, physical inactivity, supportlessness, cardiovascular deconditioning, glucose intolerance, muscle tone, day-night variations, kaliuresis 


\section{INTRODUCTION}

Spaceflight induces physiological multi-system deconditioning which may impact astronauts efficiency and create difficulties upon their return to normal gravity (Nicogossian et al., 1993). Understanding the underlying mechanisms of this process and enhancement of countermeasures remains a challenge and major priority for manned space programs. Moreover, resultant data and experience may be used to resolve common earth-based chronic healthcare problems related to increased physical inactivity, for example poststroke patients, bedridden, paralyzed or immobilized subjects, sedentary people, aging etc. Experimental opportunities during actual spaceflight being limited, the appeal of ground-based simulations is obvious and paramount (Pavy-Le Traon et al., 2007). Dry immersion (DI) is one such prolonged microgravity model. It accurately reproduces most physiological effects of microgravity, including centralization of body fluids and hypokinesia (Kozlovskaia, 2008; Navasiolava et al., 2011a; Watenpaugh, 2016). The benefit of DI, compared to more widely-known and traditional head-down bed rest (HDBR) technique, is support unloading ("supportlessness"), a state akin to weightlessness, with water hydrostatic pressure equally distributed over the body surface, providing conditions similar to complete lack of structural support (Grigor'ev et al., 2004; Navasiolava et al., 2011a). DI promotes rapid gravitational deconditioning, exceeding for some systems (i.e. for neuromuscular system) the deconditioning induced by spaceflight itself (Navasiolava et al., 2011a). However, this DI method, developed and widely used in Russia, is not yet routine elsewhere. Moreover, all DI experiments performed until now included a short daily raise for personal hygiene procedures and weighing (Navasiolava et al., 2011a). Importantly, literature shows that this type of short daily orthostatic stimulation could act as a countermeasure (Greenleaf, 1984; Vernikos et al., 1996). Therefore, in order to eliminate this aberration, our novel DI protocol did not permit subjects to rise at all for 3 days, and $a-6^{\circ}$ head down position was maintained when the subjects were out of water, as is observed in strict bedrest protocols. Hence, this study is the first DI protocol specially conceived to assess integrative aspects of "strict" DI impact.

We aimed to study integrative response to 3-day strict DI, and to assess the extent of multi-system deconditioning with regard to cardiovascular, metabolic, muscular system, daynight changes in renal excretion, and adaptive capacities/ stress effect.

\footnotetext{
Abbreviations: ALT, Alanine Aminotransferase; aMT6s, 6-sulphatoxymelatonin; ANOVA, analysis of variance; AST, Aspartate Aminotransferase; AUC, area under curve; B, baseline; BMI, body mass index; BNP, blood natriuretic peptide; BR, bed rest; DI, dry immersion; DPV (\%), plasma volume percent change; GGT, Gamma-Glutamyl Transferase; Hb, hemoglobin; Hct, hematocrit; HDBR, head down bed rest; HOMA-IR, homeostasis model assessment-insulin resistance index; HR, heart rate; hs-CRP, high-sensitivity C-reactive protein; LBNP, lower body negative pressure; $\mathrm{MCHC}$, mean corpuscular hemoglobin concentration; MCV, mean corpuscular volume; R, recovery; RBC, red blood cells; SV, stroke volume; $\dot{\mathrm{V}}_{2}$ max, maximal oxygen uptake.
}

\section{MATERIALS AND METHODS}

A total of twelve healthy non-athletic men aged 26 to 39 year. o. (age $32 \pm 1.4 \mathrm{yr}$, weight $75 \pm 2 \mathrm{~kg}$, height $178 \pm 2 \mathrm{~cm}$, BMI $23.6 \pm 0.4 \mathrm{~kg} / \mathrm{m}^{2}$, maximal oxygen uptake $\dot{\mathrm{V}}_{2} \max 39 \pm$ $1.1 \mathrm{~mL} / \mathrm{min} / \mathrm{kg}$ ) were included in the study. Subjects had no history of cardiovascular or other chronic diseases, and were not taking medication prior to the experiment. All subjects were informed about the experimental procedures and gave their written consent. The experimental protocol conformed to the standards set by the Declaration of Helsinki and was approved by the local Ethic Committee (CPP Sud-Ouest Outre-Mer I, France) and French Health Authorities ( ${ }^{\circ}$ ID RCB: 2014-A 00904-43).

\section{General Protocol}

The study was conducted at the MEDES space clinic, Toulouse, France. The experimental protocol lasted for 8 days: 3 days of ambulatory baseline measurement before immersion (B-3, B-2, B-1), 3 days (72 h) of dry immersion (DI1, DI2, DI3) and 2 days of ambulatory recovery $(\mathrm{R} 0, \mathrm{R}+1)$. Two subjects in two separate baths underwent DI simultaneously. Thermoneutral water temperature $\left(32.5-33.5^{\circ} \mathrm{C}\right)$ was continuously maintained. Light-off period was set at 23:00-07:00. General discomfort and lumbar pain under immersion were evaluated using a visual analog scale. Daily hygiene, weighing and some specific measurements required extraction from the bath. During these short out-of-bath periods, subjects maintained the $-6^{\circ}$ headdown position. Total out-of-bath supine time for the $72 \mathrm{~h}$ of immersion was $4.7 \pm 0.16 \mathrm{~h}$. Otherwise, during DI, subjects remained immersed in a supine position for all activities and were continuously observed by video monitoring. Blood pressure, heart rate (HR) and tympanic body temperature were measured twice daily at 07:00 and 19:00. Body weight was measured daily at 07:00. Onset and end of immersion both occurred at 09:00, therefore morning measurements on DI1 were performed before immersion, and on day R0-still under immersion. Before, during and after DI, water intake was ad libitum (measured), and diet was the same for all participants and standardized to body weight in energy and nutrients. Daily caloric intake was approximately 2,820 kcal for baseline and recovery and 2,270 kcal for the immersion period. Daily intake for sodium and potassium was approximately 3-4 g. Daily nutrition is detailed in supplementary data (Table S1). The timeline schematic of the global protocol is outlined in Figure 1.

This 3-day DI allowed for several protocols on different domains performed by 8 research groups. Some studies have already been published to-date (Treffel et al., 2016a,b; Arbeille et al., 2017; Demangel et al., 2017).

\section{Lower Body Negative Pressure (LBNP)-Tilt Test}

Tilt testing with combined lower body negative pressure (LBNP) was chosen as the accepted standard for measuring orthostatic tolerance (Protheroe et al., 2013). The measurement was conducted in the morning in a temperature-controlled room $(22$ $\pm 0.6^{\circ} \mathrm{C}$ ) at baseline on B-2 and immediately following DI on R0 


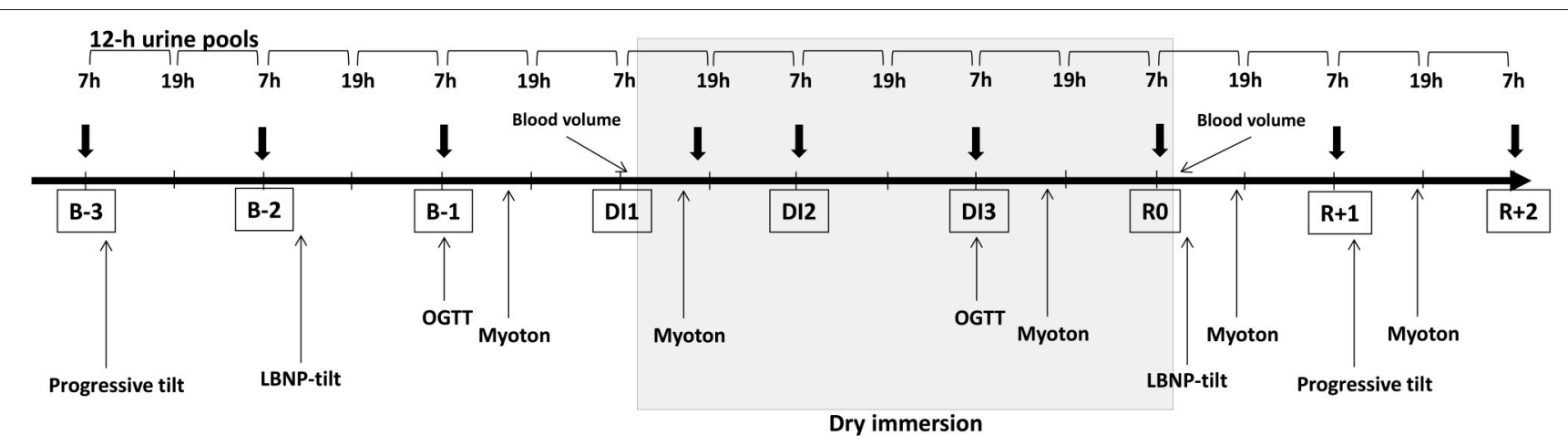

FIGURE 1 | Global protocol timeline. Thick arrows stand for blood sampling. OGTT, Oral Glucose Tolerance Test; LBNP, Lower Body Negative Pressure; B-3, B-2, B-1, days before dry immersion; DI1, DI2, DI3, first, second and third days of dry immersion. R0, R+1, R+2-days after dry immersion.

(first rising after DI). The subject remained supine for $20 \mathrm{~min}$, after which supine data were recorded for $5 \mathrm{~min}$. The tilt-table was then rotated to $80^{\circ}$ for $15 \mathrm{~min}$. After that, LBNP was applied with steps of $-10 \mathrm{mmHg}$ every $3 \mathrm{~min}$. The test was stopped at LBNP $-60 \mathrm{mmHg}$ or earlier upon appearance of pre-syncopal signs, request to stop, systolic blood pressure $\leq 80 \mathrm{mmHg}, \mathrm{HR}<$ $50 \mathrm{bpm}$ or $>170 \mathrm{bpm}$.

During the LBNP-tilt test, finger blood pressure (Nexfin, BMeye, USA) and standard ECG (Biopac, ECG 100C, USA) were recorded continuously. Orthostatic tolerance time, heart rate (HR), blood pressure (systolic, diastolic), stroke volume (SV), total peripheral resistance were estimated. Stroke volume and total peripheral resistance were evaluated from the blood pressure wave using the modelflow ${ }^{\circledR}$ method (Beatscope ${ }^{\circledR}$ software, TNO, the Netherlands). The state of autonomic nervous system was estimated via power spectrum analysis of heart rate variability. Sympathetic index, an indicator of sympatho-vagal balance, was calculated as the ratio of low-to-high frequency spectral power. Spontaneous baroreflex sensitivity was estimated using online software (televasc.fr).

\section{Progressive Tilt Test}

Progressive tilt was used to progressively stimulate the cardiovascular system (volume receptors for low angles and baroreceptors for higher angles) and vestibular system. A head-up tilt test with progressive angles $\left(0,20,45\right.$, and $\left.80^{\circ}\right)$ was performed at baseline on B-3 and on the second recovery day $(\mathrm{R}+1,24 \mathrm{~h}$ following the completion of the DI protocol). After $10 \mathrm{~min}$ of supine rest recording, the subject was tilted at $20^{\circ}$ $(5 \mathrm{~min}), 45^{\circ}(5 \mathrm{~min})$ and $80^{\circ}(5 \mathrm{~min})$, and then returned to the horizontal position.

During the progressive tilt test, HR, systolic and diastolic blood pressure, total peripheral resistance, stroke volume, sympathetic index, spontaneous baroreflex sensitivity were calculated and recorded.

\section{Blood Studies}

Antecubital venous blood samples were collected before (B-3, B2, B-1), during (DI1-evening, DI2, DI3, R0) and after DI $(\mathrm{R}+1$,
$\mathrm{R}+2$ ). Blood sampling was performed in the morning before breakfast, except for DI1 when it was done in the evening (10h of DI).

Plasma and serum samples were analyzed for electrolytes $(\mathrm{Na}+, \mathrm{K}+, \mathrm{Cl}-)$, glucose, proteins, albumin, urea, and creatinine concentrations, high-sensitivity CRP, insulin, leptin, triglycerides, total cholesterol, and HDL-cholesterol. LDLcholesterol was calculated using the Friedewald formula. Homeostasis model assessment-insulin resistance index (HOMA-IR) was calculated as fasting insulin concentration $(\mu \mathrm{U} / \mathrm{mL}) \times$ fasting glucose concentration $(\mathrm{mmol} / \mathrm{L}) / 22.5$.

Additionally, on B-3 and $\mathrm{R}+1$ blood count, GGT, ALT, AST, alkaline phosphatase, total bilirubin and prothrombin time were assessed. $\mathrm{Hb}, \mathrm{Hct}$, renin, aldosterone and blood natriuretic peptide (BNP) were assessed on $\mathrm{B}-3, \mathrm{R} 0$ and $\mathrm{R}+1$.

\section{5-g Oral Glucose Tolerance Test}

Glucose tolerance tests were performed in the morning on B-1 and DI3 (48 h of immersion). Blood glucose and insulin were measured after an overnight fast before and 30, 60, 90, and $120 \mathrm{~min}$ after a $75-\mathrm{g}$ glucose intake (consumption of glucose solution drink within $5 \mathrm{~min}$ ).

The total area under the blood glucose curve (AUC-Glu) and insulin curve (AUC-Ins) during the 75-g oral glucose tolerance test were calculated using the trapezoidal rule.

\section{Urine Sampling}

Urine pools were collected over $12 \mathrm{~h}$ (07:00-19:00 pool for "day" and 19:00-07:00 pool for "night") throughout the protocol. Light on/off periods were not taken into account for urine collection, therefore "night" pools included $4 \mathrm{~h}$ of light-on (07:00-23:00) and $8 \mathrm{~h}$ of light-off (23:00-07:00). Urine volume was measured, and aliquots stored at $-80^{\circ} \mathrm{C}$. Partial water balance, defined as the difference between consumed water and urine volume, was calculated. Urine samples were analyzed for electrolytes $\left(\mathrm{Na}^{+}\right.$, $\mathrm{K}^{+}$), cortisol and 6-sulphatoxymelatonin (aMT6s, a hepatic metabolite of melatonin usually used as a proxy measure of melatonin level). 


\section{Biochemical Analyses}

Active renin analysis was performed using a chemiluminescence immunoassay on the Liaison analyzer (DiaSorin). Plasma aldosterone was determined by a competition radioimmunoassay using a commercially available RIA kit (Immunotech, Beckman Coulter). Urinary cortisol and aMT6s assays were carried out by radioimmunoassay. All other variables from blood and urine samples were evaluated using the Architect c16,000 automated clinical chemistry analyzer (Abbott). Minimal detectable levels for aldosterone and BNP were $10 \mathrm{ng} / \mathrm{L}$, for hs-CRP-0.1 mg/L. Results that were less than the minimal detectable level, were taken for half minimal value.

\section{Blood and Plasma Volume Measurement}

Blood volume and plasma volume were estimated using the optimized CO-rebreathing method (Schmidt and Prommer, 2005) in the morning before breakfast on DI1 just before the onset of immersion and on R0 immediately at the end of immersion, in supine position. Additionally, percent change in plasma volume on $\mathrm{R} 0$ and $\mathrm{R}+1$ vs. $\mathrm{B}-3$ was calculated using $\mathrm{Hb}$ and Hct count (Dill and Costill formula): DPV $(\%)=100 \times[\mathrm{HbB}(1-0.01 \mathrm{Hcti})] /[\mathrm{Hbi}(1-0.01 \mathrm{HctB})]-$ 100 , where $\mathrm{HbB}$ and $\mathrm{HctB}$ are baseline $\mathrm{Hb}$ and Hct levels, and $\mathrm{Hbi}$ and Hcti are $\mathrm{Hb}$ and Hct on days $\mathrm{R} 0$ and $\mathrm{R}+1$, respectively.

\section{Muscle Tone}

Mechanical characteristics of muscles were determined using a hand-held myotonometer (MyotonPRO; Myoton Ltd, Estonia) before (B-1), $6 \mathrm{~h}$ following onset (DI1) and on day 3 of immersion (DI3), and in recovery period on R0 ( $6 \mathrm{~h}$ following the end of DI) and on $\mathrm{R}+1$.

During the tests, the subject was out-of-water in a relaxed supine position (dorsal and ventral decubitus). The testing end of the myotonometer was kept perpendicular to the muscle's projection in the middle of the muscle belly, at the same muscle point throughout the study. The device was used in multiscan mode, where one measurement corresponded to the mean of 5 mechanical taps. Leg measurements were taken on the left $m$. rectus femoris, $m$. tibialis anterior, $m$. gastrocnemius lateralis and $m$. soleus. Trunk measurements were taken on the right and left $m$. splenius, $m$. trapezius, $m$. longissimus cervicis, $m$. longissimus thoracis and lumbar portion of $m$. multifidus. Since trunk data showed no difference between the right and left sides (all $p>0.05$ ), results from right and left sides were averaged. For technical reasons, the data of 2 subjects (subjects $C$ and D) have not been analyzed.

\section{Statistical Analysis}

The values are presented as mean \pm SEM. Statistical analysis was performed with Prism6 GraphPad. Data were compared by a one-way and two-way repeated measures ANOVA followed by post hoc Bonferroni test. Relationships between data were examined using the Pearson correlation coefficient (r). Detailed correlations are given in supplemental data (Table S2).

\section{RESULTS}

\section{General Data}

$\mathrm{HR}$, blood pressure and body temperature remained within normal limits throughout the protocol. Baseline values of blood pressure and temperature were slightly higher in the evening. On the third day of immersion HR, blood pressure and body temperature did not differ significantly from B-1 baseline level (Figure 2). Body weight had decreased by $1-2 \mathrm{~kg}$ on the third day of immersion (Table 1).

\section{Body Fluids}

\section{Diuresis, Water Intake and Partial Water Balance}

Data are shown in Table 1. Water intake set ad libitum was decreased as anticipated on DI1, due to the known reset of water balance to lower level (Navasiolava et al., 2011c), but also on DI2 and DI3. This was likely due to discomfort during subject urination in the water tank, causing a voluntary reduction of water intake. Diuresis remained unchanged on DI1 despite a $30 \%$ reduction in water intake, therefore partial water balance decreased up to $700-800 \mathrm{~mL}$ and became negative on the first day of DI. On DI2 and DI3, water intake remained approximately $30 \%$ reduced, together with decrease in diuresis. At the recovery stage, water intake returned to baseline values, while diuresis remained diminished, with a compensatory increase in water balance.

\section{Blood and Plasma Volume}

Blood volume decreased by $11 \%$ on $\mathrm{R} 0$ (from $6.45 \pm 0.20 \mathrm{~L}$ to $5.74 \pm 0.17 \mathrm{~L}, p<0.001$ ), while total $\mathrm{Hb}$ mass diminished by $3 \%$ (from $887 \pm 33 \mathrm{~g}$ to $861 \pm 33 \mathrm{~g}, p=0.009$ ). In relation to plasma volume, the CO technique showed a $16 \pm 2 \%$ decrease at DI3 compared to DI1, and the Dill and Costill estimation showed a $14 \pm 2 \%$ decrease vs. B-3. Hence, there was no significant difference between these two types of measurements. There were no changes in plasma volume at $\mathrm{R}+1$ compared to baseline. Preimmersion plasma volume expressed in $\mathrm{ml} / \mathrm{kg}$ correlated with initial $\dot{\mathrm{V}}_{2} \max$ (Pearson $r=0.67 ; p=0.017$ ), and percent reduction in plasma volume also correlated with initial $\dot{\mathrm{VO}}_{2} \max$ (Pearson $r=-0.76$; $p=0.006$ ), i.e., fitter subjects had greater relative plasma volume at baseline and greater hypovolemia under DI.

\section{Volume-Regulating Hormones}

\section{(Renin-Angiotensin-Aldosterone System and BNP)}

A $25 \%$ increase in renin was observed prior to the end of DI (morning of R0). Twenty-four hours after DI3, renin was expectedly increased by two-fold, and aldosterone by 3 -fold. Brain Natriuretic Peptide (BNP) was below the minimum detectable level in all subjects on DI3 and $\mathrm{R}+1$ compared to 8.3 $\pm 1.6 \mathrm{ng} / \mathrm{L}$ on B-3 before DI (Table 2 ).

\section{Cardiovascular Deconditioning Orthostatic Tolerance}

Presyncopal LBNP-tilt test revealed a pronounced decrease in orthostatic tolerance with orthostatic tolerance time drop from $27 \pm 1$ min-on baseline to $9 \pm 2$ min-on R0. Before DI all subjects tolerated the first stage of test which 

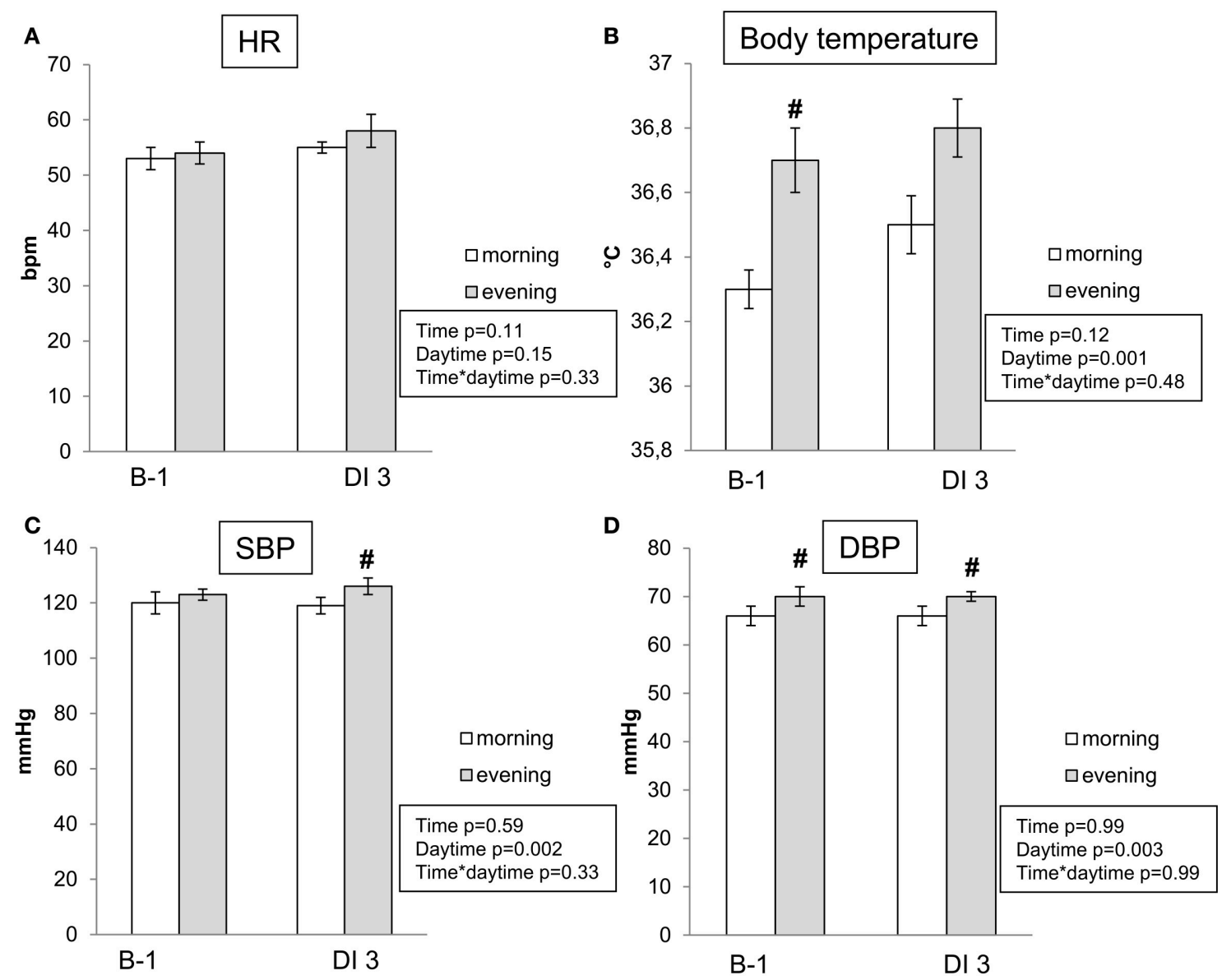

FIGURE 2 | Morning and evening heart rate (A), body temperature (B), systolic (C), and diastolic (D) blood pressure before DI and on the 3rd day of DI. Data are mean \pm SEM. No significant difference on DI3 vs. B-1. \#p $\leq 0.05$ vs. Morning.

TABLE 1 | Body weight, water intake, diuresis, and partial water balance.

\begin{tabular}{|c|c|c|c|c|c|c|c|}
\hline & B-1 & DI1 & DI2 & DI3 & Ro & $R+1$ & $p$-value (RM ANOVA) \\
\hline Morning weight, kg & $75.4 \pm 2.1$ & $74.9 \pm 2.1^{\star}$ & $73.8 \pm 2.0^{\star}$ & $73.7 \pm 2.0^{\star}$ & $73.7 \pm 2.0^{\star}$ & $74.2 \pm 2.1^{\star}$ & $<0.001$ \\
\hline Water intake, ml & $2,975 \pm 115$ & $2,181 \pm 138^{\star}$ & $2,295 \pm 184^{*}$ & $2,072 \pm 93^{\star}$ & $2,994 \pm 135$ & $3,069 \pm 158$ & $<0.001$ \\
\hline Diuresis, ml & $2,347 \pm 157$ & $2,343 \pm 180$ & $1,860 \pm 150$ & $1,478 \pm 117^{\star}$ & $1,356 \pm 76^{\star}$ & $1,763 \pm 162^{\star}$ & $<0.001$ \\
\hline Partial water balance, $\mathrm{ml}$ & $628 \pm 74$ & $-162 \pm 134^{*}$ & $435 \pm 88$ & $594 \pm 85$ & $1,638 \pm 114^{\star}$ & $1,306 \pm 97^{*}$ & $<0.001$ \\
\hline
\end{tabular}

Values are mean $\pm S E M ;{ }^{*} p \leq 0.05$ vs. $B-1$

consisted of an $80^{\circ}$ tilt for $15 \mathrm{~min}$. However, immediately after DI, 9 subjects out of 12 were unable to accomplish this tilt. Therefore, orthostatic tolerance time for non-finishers averaged at $4.95 \pm 0.7 \mathrm{~min}$. No significant correlation between percent decrease in plasma volume and post-immersion orthostatic tolerance time was found (Pearson $r=-0.36$; $p=$ $0.28)$.

Tolerance for different LBNP steps is shown in Figure 3. Before DI, all subjects finished $10 \mathrm{mmHg}$ LBNP step, a half tolerated $40 \mathrm{mmHg}$ step, and one (subject E) accomplished the last step of $60 \mathrm{mmHg}$. After DI, only 2 of the 12 subjects finished the first LBNP step, and only 1 subject (subject E) finished the step of $20 \mathrm{mmHg}$. On $\mathrm{R}+1$, all subjects finished the progressive tilt test.

\section{Hemodynamic and Autonomic Responses to LBNP-Tilt and Progressive Tilt}

Hemodynamic and autonomic responses to LBNP-tilt and Progressive tilt tests are presented in Figures 4, 5. Pre-immersion supine baseline for diastolic blood pressure, HR, total peripheral resistance and sympathetic index was greater for LBNP-tilt compared to progressive tilt, most likely due to stress caused by using the LBNP equipment. Before DI, upright position provoked expected changes in central hemodynamics and 
TABLE 2 | Blood assessment (chemistry, cardiovascular hormones, metabolic parameters, blood count).

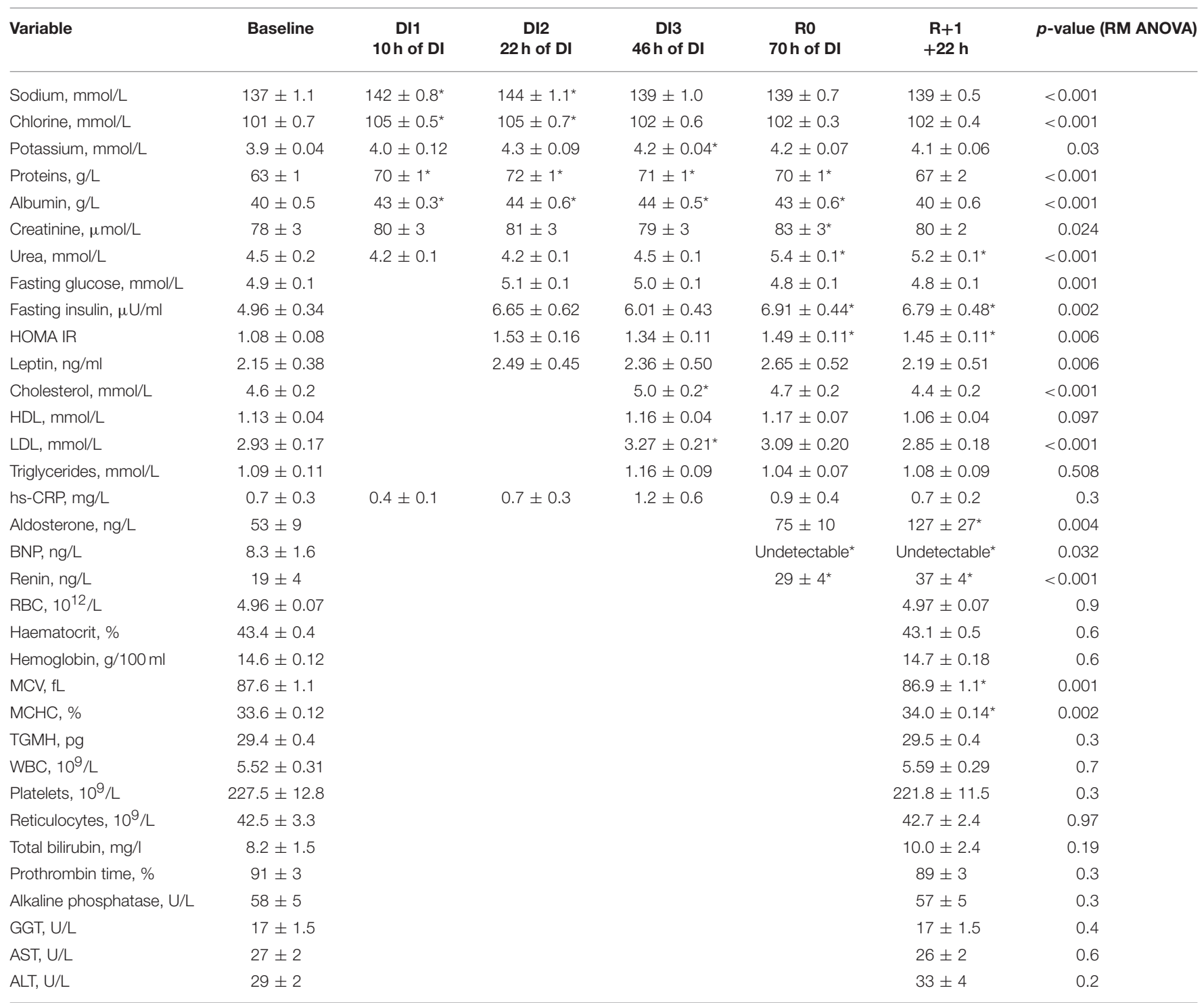

Values are mean $\pm S E M ;{ }^{*} p \leq 0.05$ vs. baseline.

cardiac autonomic neural control (increased blood pressure, $\mathrm{HR}$, total peripheral resistance and sympathetic index; decreased stroke volume and baroreflex sensitivity). These changes were progressive with progressive tilt.

Post-immersion supine measurements on R0 showed significant increases in diastolic blood pressure, HR and total peripheral resistance, a 2-fold increase in sympathetic index, and a decrease in stroke volume and baroreflex sensitivity. On $\mathrm{R}+1$, supine measurements did not differ significantly from pre-immersion levels.

Upright measurements showed a decrease in systolic blood pressure, stroke volume and baroreflex sensitivity, accompanied by pronounced tachycardia on R0. Total peripheral resistance and sympathetic index, which were already increased in supine, failed to further increase with orthostasis. On $\mathrm{R}+1$, upright position was still accompanied by greater tachycardia. Interestingly, while tilt-induced sympathetic activation before DI increased progressively with verticalisation [reaching maximum at maximal angle of tilt $\left(80^{\circ}\right)$ ], on $\mathrm{R}+1$ the maximal sympathetic index was observed at $45^{\circ}$ (difference between angles 45 and $80^{\circ}$ before DI, $p=0.07$; after DI, $p=0.8$ ).

\section{Metabolism}

\section{Blood Variables Relevant to Metabolism}

All fasting blood variables relevant to metabolism remained within physiological limits (Table 2). DI increased insulin levels, while insulin resistance (HOMA-IR) increased by $43 \pm 11 \%$ on R0 vs. baseline. Total cholesterol and LDL cholesterol fraction were moderately increased under immersion. Triglycerides and HDL remained nearly unchanged. 


\section{Number of finishers}

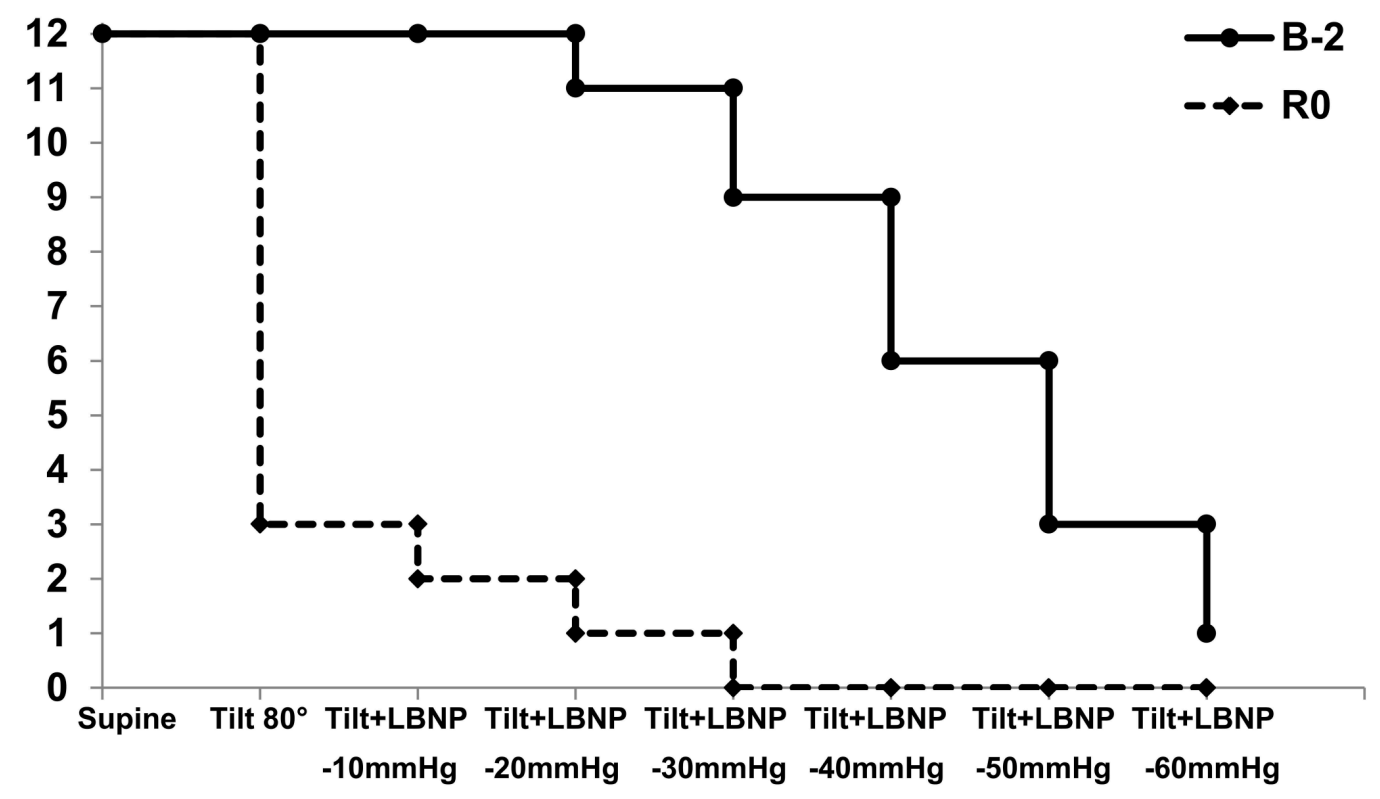

FIGURE 3 | Number of finishers for different stages of LBNP-tilt test before and immediately after 3-day DI.

\section{Oral Glucose Tolerance Test}

The response to oral glucose tolerance test is shown in Figure 6. Fasting glucose was beneath the pre-diabetic threshold $(6.1$ $\mathrm{mmol} / \mathrm{L}$ ) in all volunteers, and was not altered by DI. Glucose tolerance was compromised by DI in 11 subjects out of 12 , and in 3 subjects this impaired glucose tolerance reached pre-diabetic level (more than $7.8 \mathrm{mmol} / \mathrm{L} 2 \mathrm{~h}$ after having a glucose drink). Net insulin response (AUC-Ins) increased by $72 \pm 23 \%$ on DI3 compared to baseline. There was also a $14 \pm 5 \%$ increase in the net glucose response (AUC-Glu), while incremental AUC for glucose response increased twofold.

\section{Muscle Tone}

Muscle tone variations under DI are shown in Figure 7. Global leg muscle tone, proxy measured by "frequency" parameter, was decreased by approximately $10 \%$ under immersion. This decrease was immediate (seen $6 \mathrm{~h}$ following the onset of DI) and especially pronounced for $m$. rectus femoris (results for our subjects are also outlined in Demangel et al., 2017). The tone of $m$. gastrocnemius lateralis significantly decreased on DI3. The tone of superficial muscles of the neck and upper trunk ( $m$. trapezius and $m$. splenius) was not significantly modified under immersion. Behavior of deep back muscle tone had cervicolombar gradient. Tone immediately dropped in the upper part ( $12 \%$ for $m$. longissimus cervicis), shifting to slight decrease on D3 in the middle ( $m$. longissimus thoracis) and was not modified in the lower part (lumbar portion of $m$. multifidus). Six hours following the end of DI, muscle tone was completely restored.

\section{Day-Night Variations in Urinary Excretion Urinary Sodium}

At baseline, daytime excretion exceeded night-time excretion by approximately $20 \%$. On DI3, day-night variations in $\mathrm{Na}^{+}$ excretion were preserved (Figure 8A).

\section{Urinary Potassium}

At baseline, day-night differences in $\mathrm{K}^{+}$excretion were clearly marked and stable, with 2.5 fold greater excretion in daytime. There was no effect on day-night difference in $\mathrm{K}^{+}$excretion after DI1. At the start of DI2, night excretion tended to increase while day excretion tended to decrease, showing an important drop in day-night difference reaching significance on R0 (Figure 8B).

Interestingly, $24 \mathrm{~h}$ excretion was stable for $\mathrm{K}^{+}$throughout the protocol, whereas $24 \mathrm{~h} \mathrm{Na}^{+}$excretion varied significantly within immersion, peaking at the onset of about $40 \%$ over baseline and drop at the end of DI of about $60 \%$ beneath baseline.

\section{Urinary Cortisol}

Urinary cortisol had pronounced day-night oscillations. Cortisol levels tended to decrease in the first $10 \mathrm{~h}$ of DI, then to increase on the night of DI1, however, these changes were within physiological limits. Day-night differences in cortisol excretion were smoothed at the onset of immersion, but restored on DI2. Cortisol level recovered immediately after DI (Figure 9A).

\section{Urinary aMT6s}

Urinary aMT6s had pronounced day-night oscillations, which tended to accentuate during immersion mainly due to the increased night release. On R0, day-night differences in aMT6s 


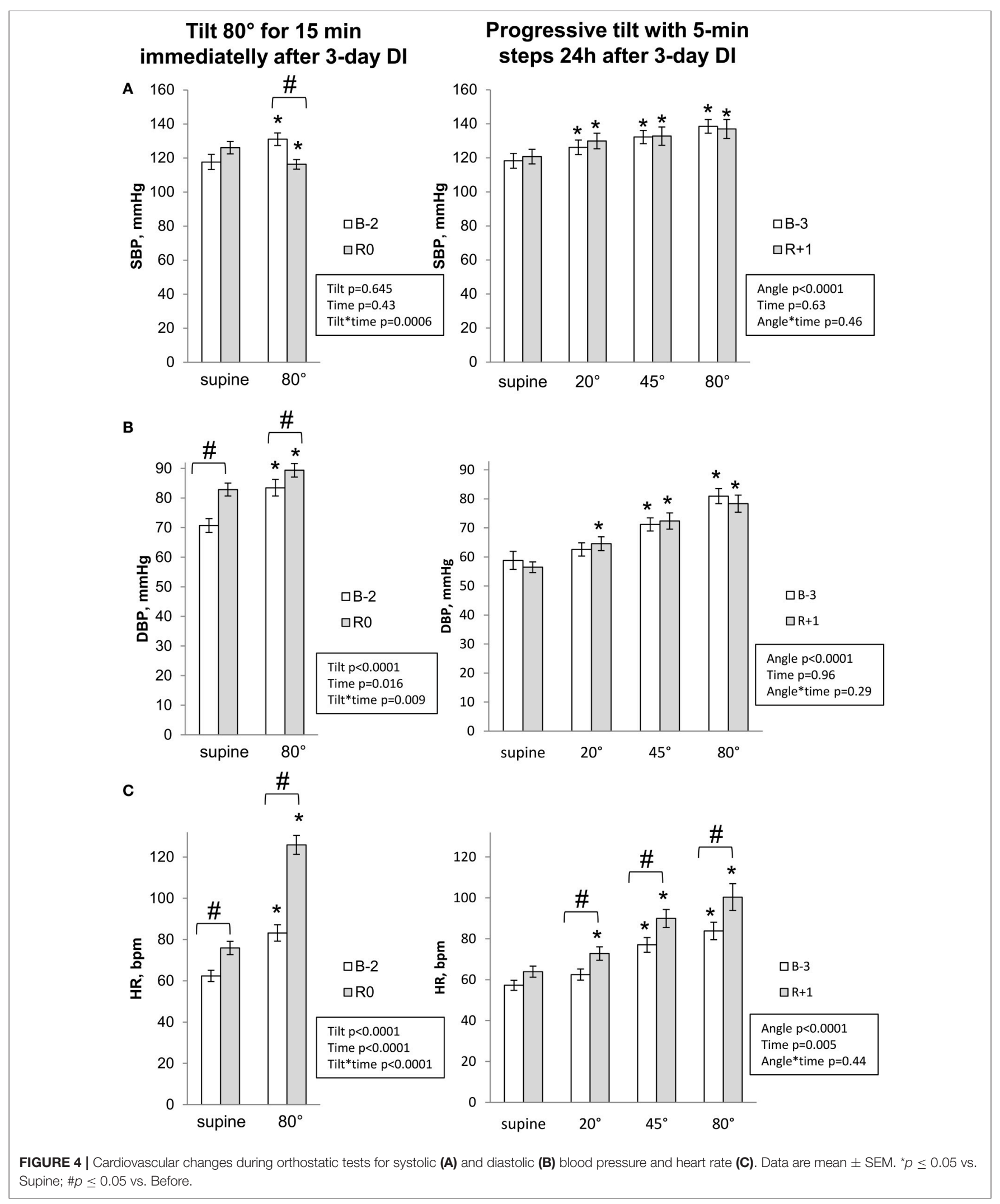




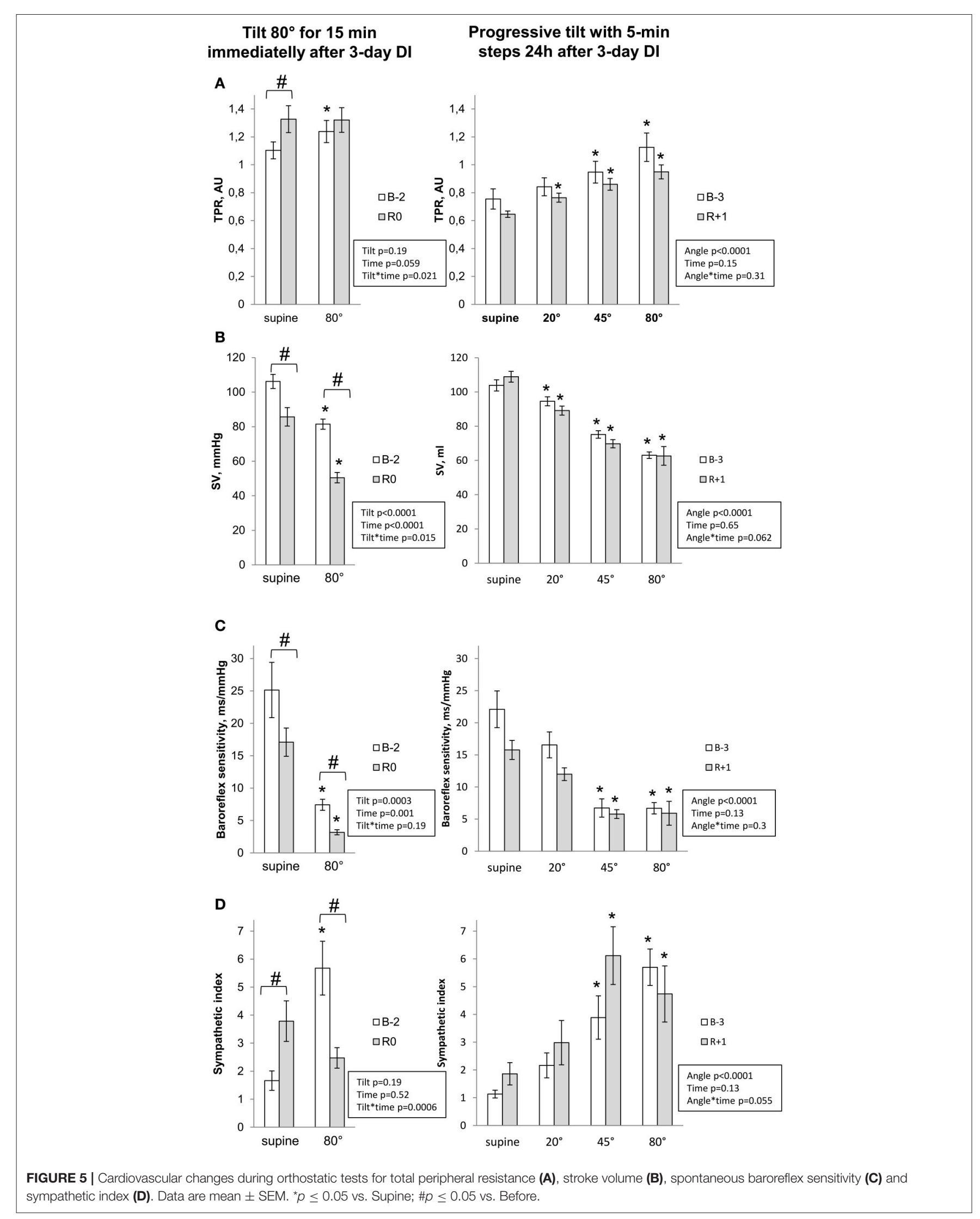



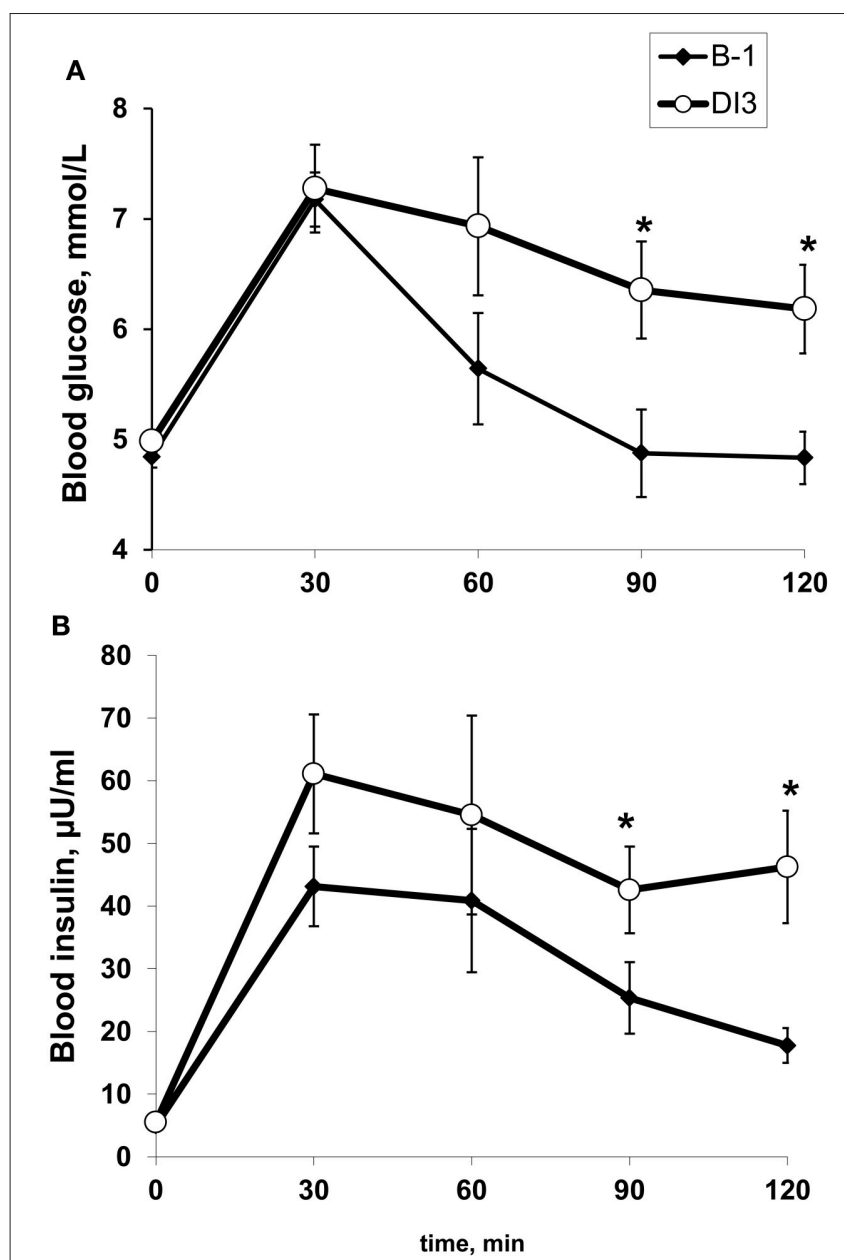

FIGURE 6 | The effect of 48-h dry immersion on the glucose (A) and insulin (B) response to 75-g oral glucose tolerance test in 12 healthy subjects. Data are mean \pm SEM. ${ }^{*} p \leq 0.05$ vs. B-1.

secretion were diminished, suggesting readaptation to normal conditions (Figure 9B).

\section{General Discomfort and Pain during DI}

In general, the majority of subjects described the discomfort level experienced under DI as 30-45 out of 100. Some subjects reported pronounced discomfort at nighttime. We observed important inter-subject variance in auto-reported discomfort level, varying from 0 to 88 . There was no significant difference in discomfort level between the different days of DI. Eleven out of the 12 subjects reported moderate back pain under DI, which was predominantly localized in the lumbar area for 10 subjects. Lumbar pain intensity as estimated on DI2 was $3.8 \pm 0.7$ on the scale of 1-10. There was a positive correlation between lumbar pain intensity and mean 24-h urinary cortisol during DI (199 \pm $24 \mathrm{mmol} / 24 \mathrm{~h}$ ) (Pearson $r=0.64 ; p=0.02$ ). As well, we observed a positive correlation between mean discomfort level and mean 24-h urinary melatonin metabolite under DI ( $40.5 \pm 5.4 \mu \mathrm{g} / 24 \mathrm{~h})$ (Pearson $r=0.67 ; p=0.018$ ).

\section{Blood Electrolytes, Blood Count, Liver-Related Biochemistry, hs-CRP}

Data are detailed in Table 2. Plasma electrolytes showed modest changes without clinical relevance. Blood count remained unchanged except for a modest but highly significant decrease in $\mathrm{RBC}$ volume, accompanied by slight increase in $\mathrm{MCHC}$ on $\mathrm{R}+1$ vs. B-3. Blood GGT, ALAT, ASAT, alkaline phosphatase, total bilirubin, prothrombin time and serum albumin were not modified on $\mathrm{R}+1$ vs. B-3. CRP levels were unaltered by DI.

\section{DISCUSSION}

\section{Main Findings}

Orthostatic tolerance time dropped from $27 \pm 1$ min to $9 \pm$ 2 min after DI. Impairment in glucose tolerance was significantly pronounced. Net insulin response increased by $72 \pm 23 \%$ on DI3 compared to baseline. Global leg muscle tone was reduced by approximately $10 \%$ with the DI protocol. Day-night changes in temperature, heart rate and blood pressure were preserved on the third day of DI. Day-night levels of urinary $\mathrm{K}^{+}$were reduced, beginning on the second day of immersion, while 24-h $\mathrm{K}^{+}$excretion remained stable. Urinary cortisol and melatonin metabolite levels increased with DI, although within normal limits. A positive correlation between lumbar pain intensity estimated on DI2 and mean 24-h urinary cortisol under DI was observed.

\section{Need for Strict DI without Daily Rise}

In the present study, we observed pronounced cardiovascular deconditioning after 3 days of strict DI. This deconditioning was characterized by a predisposition to orthostatic intolerance and tachycardia.

\section{Orthostatic Intolerance}

Seventy-five percent of our subjects were intolerant to orthostasis on R0 after 3 days of strict DI. In comparison with literature data using chi-square test, orthostatic intolerance rate after strict bed rest without countermeasures does not differ significantly from our findings: $5 / 11(45 \%)$-after 4 days $(p=0.15), 4 / 6(67 \%)$-after 14 days $(p=0.7), 5 / 9(56 \%)$-after 28 , or 30 days $(p=0.35)$, and 4/7 (57\%)-after 42 days of HDBR ( $p=0.4$ ) (Pavy-Le Traon et al., 1999).

However, the reported rate of orthostatic intolerance following non-strict DI seems much less-approximately 15 to $40 \%$ of subjects for 1 to 7 days of DI. Indeed, after $24 \mathrm{~h}$ of DI, 2 subjects out of 10 were reported to be intolerant to the orthostatic test on R0 ( $p=0.01$ ), after 1.5 days of DI- 1 out of 6 ( $p=0.02$ ) (Navasiolava et al., 2011a), after 3 days of DI-1 out of $6(p=0.02)$ (Iwase et al., 2000) and 0 out of $4(p=0.009)$ (Miwa et al., 1997), after 5-day DI-4 out of 7 ( $p=0.4$ ) (one was already intolerant before DI) (Coupé et al., 2013), and after 7 days of DI-2 out of $6(p=0.09)$ (Iarullin et al., 1987) and 1 out of $8(p=0.006)$ (Navasiolava et al., 2011b). The main differentiator of our DI was the maintenance of supine position throughout. Our data suggest that daily short periods out of the water tank in sitting or standing positions represent a powerful countermeasure against orthostatic intolerance induced by 

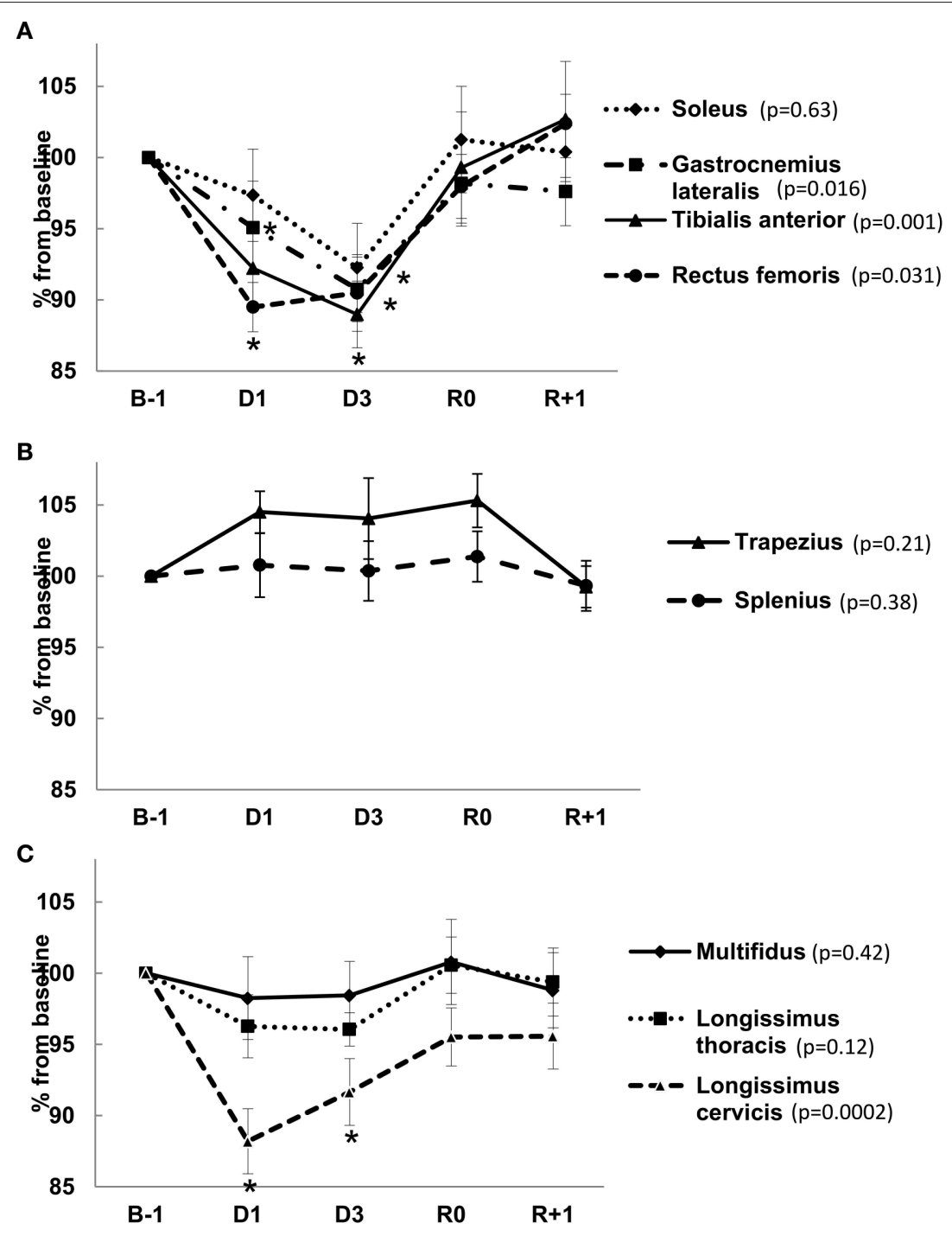

FIGURE 7 | Percent change in muscle tone of leg muscles (A), superficial neck and upper trunk muscles (B) and deep back muscles (C) $6 \mathrm{~h}$ following the onset (D1), on day 3 of immersion (D3), in recovery period on $\mathrm{R} 0$ ( $6 \mathrm{~h}$ following the end of $\mathrm{DI}$ ) and on $\mathrm{R}+1$. Data are mean $\pm \mathrm{SEM}$. ${ }^{*} p \leq 0.05 \mathrm{vs}$. $\mathrm{B}-1$.

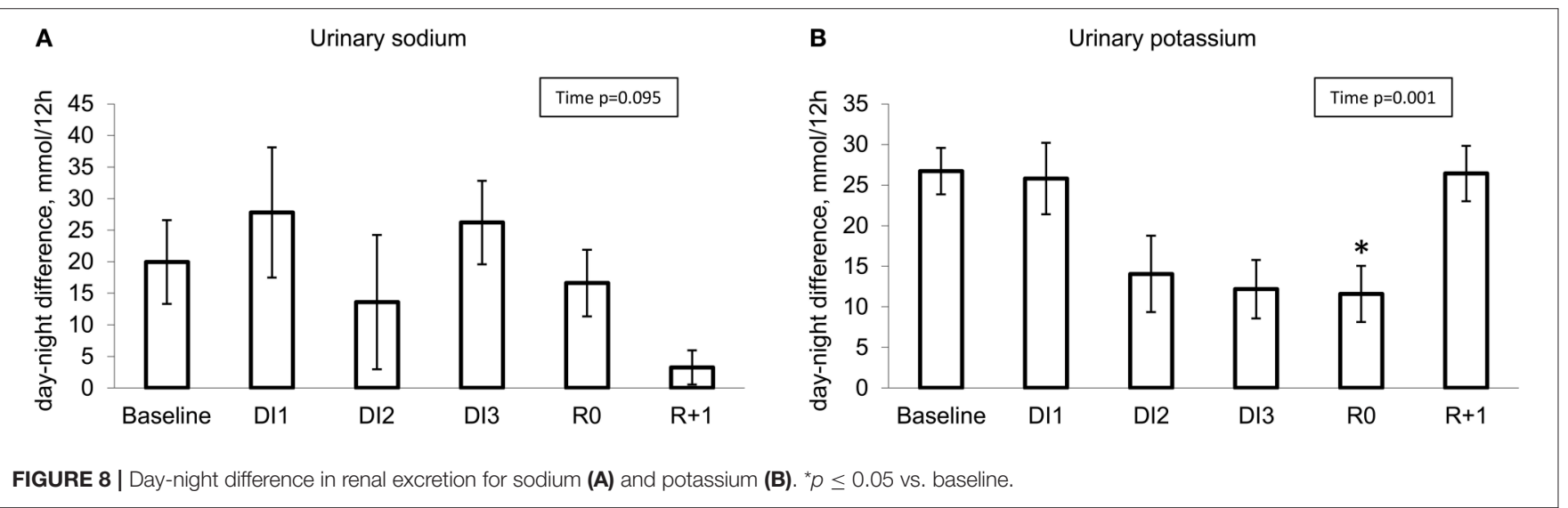




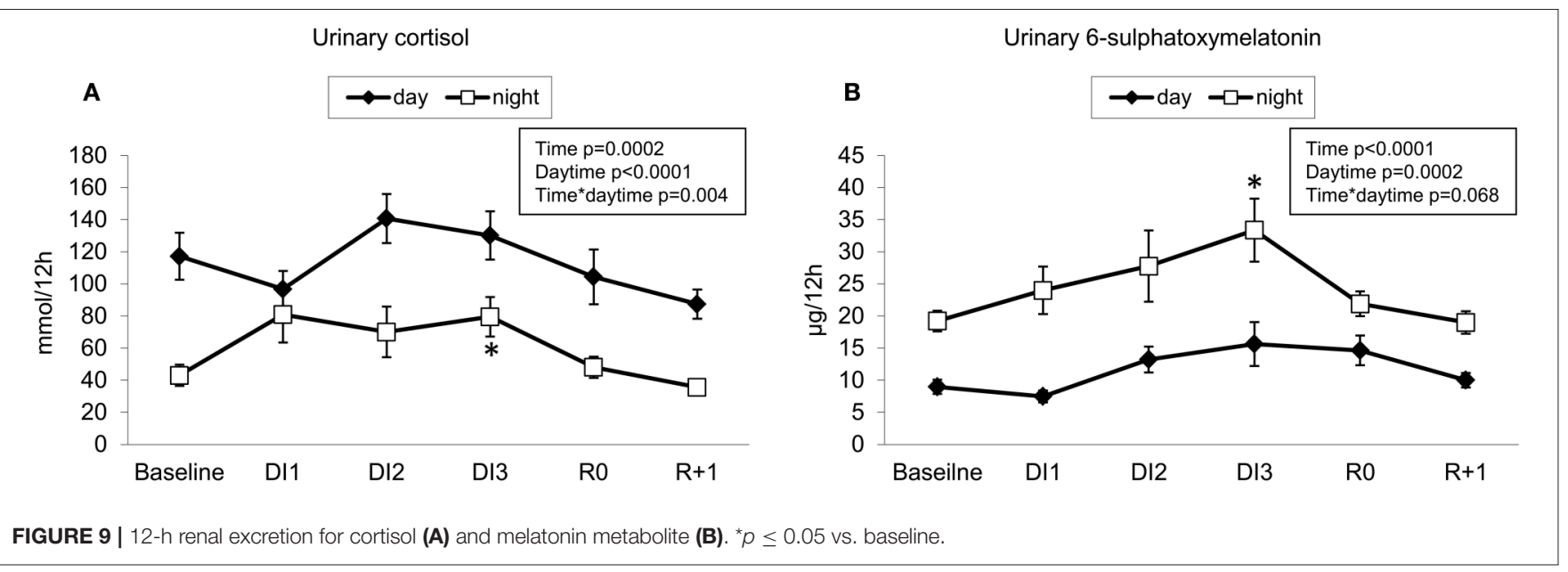

DI. Periodic short gravitational stimuli appear to be effective countermeasures, maintaining gravitational tolerance. Vernikos et al. (1996) demonstrated a very efficient preventive effect of a short period in a standing position (for $2 \mathrm{~h}$ daily) against orthostatic intolerance after 4 days of HDBR. The beneficial effect of LBNP for $20 \mathrm{~min} /$ day during both spaceflight and bed rest was discussed in a review by Clement and Pavy-Le Traon (2004). They cite the evidence that daily centrifugation for 3045 min reduces most of the physiological markers associated with orthostatic intolerance. Thus, a daily short period of orthostatic stress is sufficient to reduce the risk of orthostatic intolerance after simulated weightlessness. Daily short term gravitational load might also promote the preservation of baroreflex sensitivity and autonomic balance.

\section{Tachycardia}

Resting tachycardia measurements observed immediately after immersion at R0 suggest that even the supine position out of water represents an additional workload for subjects exposed to immersion. Strict DI induces more pronounced cardiovascular deconditioning than non-strict DI, even if daily rise is very short. Similar to our finding, an increase in both asleep and awake HR was documented following 5-to-10-day space mission (FritschYelle et al., 1996). The majority of previous DI studies did not report significant changes in resting supine HR following DI (Navasiolava et al., 2011a). Tachycardia, when measured in an upright position, demonstrated a $50 \%$ increase at the first rise following DI (125 \pm 5 bpm vs. $83 \pm 4 \mathrm{bpm})$. Previous studies reported a $25 \%$ increase in upright $\mathrm{HR}$ after 3-day DI (Iwase et al., 2000), 30\%- after 5-day DI (Coupé et al., 2013), 40\% and 48\%after 7-day DI (Iarullin et al., 1987; Navasiolava et al., 2011a), and 50\%- after 10-day DI (Panferova, 1976). Taken together, literature suggests that resting and upright tachycardia readings are emphasized and enhanced following strict DI compared to non-strict DI protocols.

\section{Sympathetic Regulation of Cardiovascular Functions} Sympathetic neural control is extremely important in maintaining blood pressure homeostasis against gravity (Mano,
2005). In our study, strict DI clearly affected cardiac sympathetic neural control and baroreflex sensitivity. Supine sympathetic index, which presumably reflects resting cardiac sympathetic activity, was more than 2-fold increased immediately after DI, suggesting an activation of sympathetic nervous system. Supine sympathetic index failed to further increase in response to tilt, unlike previous DI studies, such as that by Miwa et al. (1997), who found an increase in upright sympathetic index following 3-day DI. Observed alteration in the sympathetic index response to progressive tilt may be related to dysregulation of high-low pressure baroreceptors. Therefore, a decrease in sympathetic index may represent a factor in orthostatic intolerance. We found that upright sympathetic index on R0 tended to correlate with orthostatic tolerance time (Pearson $r=$ $0.54 ; p=0.068)$.

\section{Vasoconstriction to Orthostasis}

Total peripheral resistance is an integrative characteristic of overall resistance of peripheral vasculature in the systemic circulation. Insufficient vasoconstriction (increase in total peripheral resistance) to orthostatic stimuli is an acknowledged major factor for microgravity-induced orthostatic hypotension (Zhang, 2001). Astronauts intolerant to orthostatism fail to adequately increase TPR when upright (Buckey et al., 1996). Increased resting total peripheral resistance observed in our study suggests greater basal vasoconstriction and therefore a decrease in the reserve of vasoconstriction, with limitation of the vasoconstrictive response. According to Convertino hypothesis (Convertino, 1999), diminished vasoconstrictive reserve may be the main mechanism of vasoconstrictor insufficiency in cases of orthostatic intolerance. The maximal capacity of vasoconstriction is not altered under microgravity (Convertino, 1999), but hypovolemia might induce an increase in baseline vasoconstriction and thus decrease the vasoconstrictive reserve. However we did not observe direct correlation between upright total peripheral resistance at $\mathrm{R} 0$ and orthostatic tolerance time (Pearson $r=0.47 ; p=0.12$ ), suggesting involvement of additional factors into orthostatic intolerance. 


\section{Water-Sodium Balance and Plasma Volume}

Resetting of water-sodium balance under DI, accompanied by acute BNP increase and renin-aldosterone suppression, usually occurs over the first to second day, with a subsequent return in hormonal concentrations to their usual level (Leach Huntoon et al., 1998; Navasiolava et al., 2011a,c; Coupé et al., 2013). We did not assess the initial hormonal responses. The observed moderate rise in renin at the end of DI may be related to the slight decrease in dietary sodium under DI. However we did not find direct correlation between the decrease in dietary sodium and the increase in renin at the end of DI (Pearson $r=-0.36$; $p=0.26$ ). Similarly, Shulzhenko et al. (1980) reported a minor increase in plasmatic renin on DI7 and in aldosterone on DI5 and DI6. The observed decrease in plasma volume of $16 \%$ is in accordance with the data described in other DI studies, with 15-20\% hypovolemia (Leach Huntoon et al., 1998; Navasiolava et al., 2011a; Coupé et al., 2013). The similarity in hypovolemia numbers obtained by the CO technique and the Dill and Costill estimation suggests the accuracy of the indirect method of plasma volume estimation by $\mathrm{Hb}$ and Hct count under DI. The observed greater reduction in plasma volume in fitter subjects was in line with Convertino (1996a).

\section{What May Be Responsible for This Decrease in Orthostatic Tolerance?}

Hypovolemia appears to be the major contributor for the observed rapid cardiovascular impairment in DI. However, the degree of hypovolemia in our study is similar to that reported for non-strict DI (15\%-Leach Huntoon et al., 1998; 17-18\%-Coupé et al., 2013; 15\%-Navasiolava et al., 2011c; 12-16\%-Gogolev et al., 1980). Moreover, the percentage decrease in plasma volume did not directly correlate with orthostatic tolerance time (Pearson $r=$ $-0.36 ; p=0.28$ ). The degree of hypovolemia develops within the first $24 \mathrm{~h}$ and is not greatly dependant on the duration of actual or simulated microgravity, whereas the degree of orthostatic intolerance tends to increase with extension of microgravity. Aside from a reduction in plasma volume, other mechanisms including increased lower limb venous capacitance (Convertino, 1996b), leg muscle tone diminution, compromised sympathetic regulation (Convertino, 2002; Mano, 2005), myocardial function and baroreflex sensitivity (Engelke et al., 1996; Convertino, 2002) may also contribute to cardiovascular deconditioning following DI. Vascular impairment at macro- (Zhang, 2001) and microvascular (Coupé et al., 2009) level promoted by physical inactivity and reduced shear stress (de Groot et al., 2006), in addition to metabolic, hormonal and vestibular changes may also play a role.

\section{Rapid and Profound Metabolic Changes Induced by Strict DI}

The negative metabolic effects of DI are mainly related to increased inactivity. DI rapidly impaired glucose metabolism and lipid profile, inducing a decrease in insulin sensitivity and dyslipidemia. The same changes were observed in bed rest experiments (Blanc et al., 2000; Hamburg et al., 2007; Bergouignan et al., 2010). Even short-time physical inactivity appears sufficient to impair metabolism (Hamburg et al., 2007; Coupé et al., 2013; this study).

Some studies suggest that fasting glucose is not modified by DI, and fasting insulin is somewhat increased following 7-day DI (Navasiolava et al., 2011a). However, the effect of DI on glucose tolerance has not previously been explicitly investigated. This study is believed to be the first to examine the effect of DI on oral glucose tolerance test in depth. Data in the published literature indicate that bedrest (BR) increases insulin response to glucose loading without deterioration in glucose tolerance (Kiilerich et al., 2011-non-strict 7-day BR in men with allowed sitting 5h/day, Blanc et al., 2000-7-day strict HDBR in women, Dirks et al., 2016-7-day strict BR in men, mixed-meal tolerance test) or with slight increase in the net glucose response of about 6\% (Hamburg et al., 2007-5-day BR in men and women, Heer et al., 2014-21-day BR in men). Globally, in BR, an increased insulin response is able to prevent glucose increase, or at a minimum, this glucose increase remains relatively moderate. In our DI experiment, a shorter exposure (2 days) seems to induce a greater impairment in glucose tolerance (14\%-increase in the net glucose response). However, caution should be employed when interpreting these results as our subjects showed pre-immersion response to oral glucose intake with a faster decline in glucose concentration compared to baseline of bedrest studies reported by Heer et al. (2014) and by Hamburg et al. (2007), whereas postimmersion response was much closer to that observed after those bedrest protocols. This difference in baseline measurements may be related to individual sensitivity of subjects and limits the possibility of direct comparison.

Increased blood glucose and dyslipidemia may compromise endothelial integrity and microvascular functions (Hamburg et al., 2007; Yuan et al., 2015), thus indirectly contributing to orthostatic intolerance. However, we did not observe any direct correlation between induced glycemia on DI3 and orthostatic tolerance time following DI (Pearson $r=-0.32 ; p=0.31$ ).

\section{Day-Night Variations}

Day-night variability in HR, blood pressure and body temperature on DI3 did not differ from baseline, suggesting a good adaptation to DI conditions. Circadian rhythm of $\mathrm{K}^{+}$ excretion is one of the most consistent and stable physiological circadian fluctuations (Gumz et al., 2015). It is independent of cyclic potassium intake, adrenal hormones, changes in plasma potassium, renal nerves and sodium cyclic excretion. Physiologic circadian rhythm in potassium excretion has high amplitude and is driven by a brain oscillator. As suggested by Gumz and Rabinowitz (2013), fluctuations in potassium excretion are regulated by circadian fluctuations in $\mathrm{K}^{+}$transporter gene expression. "Predictive" signals from central nervous system (CNS) cyclically change kidney sensitivity for the excretory commands, thus preparing increase in renal excretion for the period of expected potassium intake with meal (Gumz et al., 2015).

In our study, circadian rhythm in potassium excretion was preserved, however we observed a delayed diminution in its amplitude. A possible rationale is that DI might induce a forward shift of day-night cycle (under DI the subjects have sleeping 
difficulties and might fall asleep later). Consequently, the 19:0007:00 urine pool would contain more "daily" urine than before, so night kaliuresis would appear to be elevated. However, night melatonin was not decreased as expected in case of increased "contamination" of night urine with daily portion. This suggests another potential explanation-a certain disturbance in circadian regulation itself. The observed delayed changes in renal $\mathrm{K}^{+}$ excretion variance (and delayed restoration) might reflect a change in its "predictive"/feedforward regulation by homeostatic system of CNS (Gumz et al., 2015). Other studies have also shown that circadian rhythms could be modified by spaceflight (Fuller et al., 1994; Guo et al., 2014) and bed rest (Millet et al., 2001; Pavy-Le Traon et al., 2007; Liang et al., 2012). Bed rest did not change the excretion of $\mathrm{K}^{+}$, but altered the rhythmicity and circadian amplitude of $\mathrm{Na}^{+}$excretion (Millet et al., 2001; Liang et al., 2012).

\section{Stress Is Moderate in $\mathrm{DI}$ and Might Be Related to Back Pain}

A relatively modest increase in cortisol suggests quite a moderate physiological stress effect of DI. During immersion, the increase in urinary cortisol level was more evident at night. It may be related to the greater perception of the stress factors inherent to DI at night time. Interestingly, in the first hours of immersion, a drop in cortisol levels was observed. In part, this is likely due to the fact that short time immersion is quite comfortable and relaxing, which is why the method is employed in a spa setting (Navasiolava et al., 2011a). The positive correlation between the change in urinary cortisol and self-reported lumbar pain level confirms the role of cortisol in response to stress under DI.

This lumbar pain was not accompanied by muscle spasm. Indeed, an increase in lower back muscle tone might be expected, as lumbar pain should induce a reflex contraction of the lumbar paravertebral muscles. However, direct support withdrawal immediately switches off the tone of postural muscles (Navasiolava et al., 2011a). We observed this immediate response for antigravity leg muscles and upper deep back, but not for lower deep back. Muscle tone stability in lower deep back could represent an averaged effect of immersion (which decreases the tone) and back pain (which increases tone). The deep muscles of the lower back, which play an important role in stabilizing the joints within the spine, may preserve their tone to counteract lumbar deformation associated with back pain.

\section{REFERENCES}

Arbeille, P., Avan, P., Treffel, L., Zuj, K., Normand, H., and Denise, P. (2017). Jugular and portal vein volume, middle cerebral vein velocity, and intracranial pressure in dry immersion. Aerosp. Med. Hum. Perform. 88, 457-462. doi: 10.3357/AMHP.4762.2017

Bergouignan, A., Momken, I., Schoeller, D. A., Normand, S., Zahariev, A., Lescure, B., et al. (2010). Regulation of energy balance during long-term physical inactivity induced by bed rest with and without exercise training. J. Clin. Endocrinol. Metab. 95, 1045-1053. doi: 10.1210/jc. 2009-1005
Night increase in melatonin might aim to counteract the effect of unusual environment and promote sleeping. Positive correlation between urinary melatonin and global discomfort level under DI suggests the "efforts" of melatonin to counteract discomfort.

\section{CONCLUSION}

Dry immersion (DI) induces an accelerated model of cardiovascular deconditioning in response to microgravity. The "support unloading" induced by DI provides rapid and profound cardiovascular deconditioning. This is exemplified by increased plasma volume loss, orthostatic intolerance, pronounced autonomic changes, pronounced metabolic impairment, rapid and profound decreases in muscle tone, and influence on circadian rhythms. DI is tolerated well enough despite backache, and shows rather moderate stress effect. Such rapid, profound and quickly reversible gravitational deconditioning renders the strict DI model extremely significant to test countermeasures for microgravity-induced deconditioning and physical inactivity-related pathologies.

\section{AUTHOR CONTRIBUTIONS}

Conception and design of the study: MC, NN, GG, and RM. Data acquisition and sample analysis: SD, LA, RM, VR, FL, LT, and RW. Analysis and interpretation of results, drafting and revising the article: All authors.

\section{FUNDING}

This dry immersion was supported by CNES (CNES grant number $\left.2014 \mathrm{n}^{\circ} 4800000748\right)$.

\section{ACKNOWLEDGMENTS}

We thank the volunteers and the stuff of MEDES for participation in this protocol at the MEDES space clinic in 2015.

\section{SUPPLEMENTARY MATERIAL}

The Supplementary Material for this article can be found online at: https://www.frontiersin.org/articles/10.3389/fphys. 2017.00799/full\#supplementary-material

Blanc, S., Normand, S., Pachiaudi, C., Fortrat, J. O., Laville, M., and Gharib, C. (2000). Fuel homeostasis during physical inactivity induced by bed rest. J. Clin. Endocrinol. Metab. 85, 2223-2233. doi: 10.1210/jc.85.6.2223

Buckey, J. C. Jr., Lane, L. D., Levine, B. D., Watenpaugh, D. E., Wright, S. J., Moore, W. E., et al. (1996). Orthostatic intolerance after spaceflight. J. Appl. Physiol. (1985) 81, 7-18.

Clement, G., and Pavy-Le Traon, A. (2004). Centrifugation as a countermeasure during actual and simulated microgravity: a review. Eur. J. Appl. Physiol. 92, 235-248. doi: 10.1007/s00421-004-1118-1

Convertino, V. A. (1996a). Clinical aspects of the control of plasma volume at microgravity and during return to one gravity. Med. Sci. Sports Exerc. 28, 45-52. 
Convertino, V. A. (1996b). "Exercise and adaptation to microgravity environment," in Handbook of Physiology, Environmental Physiology, eds M. J. Fregly and C. M. Blatteis (New York, NY: Oxford University Press), 815-843.

Convertino, V. A. (1999). G-factor as a tool in basic research: mechanisms of orthostatic tolerance. J. Gravit. Physiol. 6, 73-76.

Convertino, V. A. (2002). Mechanisms of microgravity induced orthostatic intolerance: implications for effective countermeasures. J. Gravit. Physiol. 9, $1-13$.

Coupé, M., Fortrat, J. O., Larina, I., Gauquelin-Koch, G., Gharib, C., and Custaud, M. A. (2009). Cardiovascular deconditioning: from autonomic nervous system to microvascular dysfunctions. Respir. Physiol. Neurobiol. 169, S10-S12. doi: 10.1016/j.resp.2009.04.009

Coupé, M., Tomilovskaya, E., Larcher, F., Diquet, B., Pastushkova, L., Kozlovskaya, I., et al. (2013). Body fluid changes, cardiovascular deconditioning and metabolic impairment are reversed 24 hours after a 5-day dry immersion. $O$. J. Neph. 3, 13-24. doi: 10.4236/ojneph.2013.31004

de Groot, P. C., Bleeker, M. W., and Hopman, M. T. (2006). Magnitude and time course of arterial vascular adaptations to inactivity in humans. Exerc. Sport Sci. Rev. 34, 65-71. doi: 10.1249/00003677-200604000-00005

Demangel, R., Treffel, L., Py, G., Brioche, T., Pagano, A. F., Bareille, M. P., et al. (2017). Early structural and functional signature of 3-day human skeletal muscle disuse using the dry immersion model. J. Physiol. 595, 4301-4315. doi: $10.1113 /$ JP273895

Dirks, M. L., Wall, B. T., van de Valk, B., Holloway, T. M., Holloway, G. P., Chabowski, A., et al. (2016). One week of bed rest leads to substantial muscle atrophy and induces whole-body insulin resistance in the absence of skeletal muscle lipid accumulation. Diabetes 65, 2862-2875. doi: 10.2337/db1 5-1661

Engelke, K. A., Doerr, D. F., Crandall, C. G., and Convertino, V. A. (1996). Application of acute maximal exercise to protect orthostatic tolerance after simulated microgravity. Am. J. Physiol. 271, R837-R847.

Fritsch-Yelle, J. M., Charles, J. B., and Jones, M. M. (1996). Microgravity decreases heart rate and arterial pressure in humans. J. Appl. Physiol. 80, 910-914.

Fuller, C. A., Hoban-Higgins, T. M., Griffin, D. W., and Murakami, D. M. (1994). Influence of gravity on the circadian timing system. Adv. Space Res. 14, 399-408. doi: 10.1016/0273-1177(94)90431-6

Gogolev, K. I., Aleksandrova, E. A., and Shul'zhenko, E. B. (1980). Comparative assessment of changes during antiorthostatic hypokinesia and immersion in man. Hum. Physiol. 6, 392-396.

Greenleaf, J. E. (1984). Physiological responses to prolonged bed rest and fluid immersion in humans. J. Appl. Physiol. 57, 619-633.

Grigor'ev, A. I., Kozlovskaia, I. B., and Shenkman, B. S. (2004). The role of support afferents in organisation of the tonic muscle system. Ross. Fiziol. Zh. Im. I. M. Sechenova. 90, 508-521.

Gumz, M. L., and Rabinowitz, L. (2013). Role of circadian rhythms in potassium homeostasis. Semin. Nephrol. 33, 229-236. doi: 10.1016/j.semnephrol.2013.04.003

Gumz, M. L., Rabinowitz, L., and Wingo, C. S. (2015). An integrated view of potassium homeostasis. N. Engl. J. Med. 373, 60-72. doi: 10.1056/NEJMra1313341

Guo, J. H., Qu, W. M., Chen, S. G., Chen, X. P., Lv, K., Huang, Z. L., et al. (2014). Keeping the right time in space: importance of circadian clock and sleep for physiology and performance of astronauts. Mil. Med. Res. 1:23. doi: 10.1186/2054-9369-1-23

Hamburg, N. M., McMackin, C. J., Huang, A. L., Shenouda, S. M., Widlansky, M. E., Schulz, E., et al. (2007). Physical inactivity rapidly induces insulin resistance and microvascular dysfunction in healthy volunteers. Arterioscler. Thromb. Vasc. Biol.. 27, 2650-2656. doi: 10.1161/ATVBAHA.107. 153288

Heer, M., Baecker, N., Wnendt, S., Fischer, A., Biolo, G., and Frings-Meuthen, P. (2014). How fast is recovery of impaired glucose tolerance after 21-day bed rest (NUC study) in healthy adults? ScientificWorldJournal 2014:803083. doi: 10.1155/2014/803083

Iarullin, K.h., Kh., Simonov, L. G., and Vtoryi, S. A. (1987). Changes in regional and central hemodynamics during a 7-day immersion in water [in Russian, English summary]. Kosm. Biol. Aviakosm. Med. 21, 45-50.

Iwase, S., Sugiyama, Y., Miwa, C., Kamiya, A., Mano, T., Ohira, Y., et al. (2000). Effects of three days of dry immersion on muscle sympathetic nerve activity and arterial blood pressure in humans. J. Auton. Nerv. Syst. 79, 156-164. doi: 10.1016/S0165-1838(99)00076-4

Kiilerich, K., Ringholm, S., Biensø, R. S., Fisher, J. P., Iversen, N., van Hall, G., et al. (2011). Exercise-induced pyruvate dehydrogenase activation is not affected by 7 days of bed rest. J. Appl. Physiol. 111, 751-757. doi: 10.1152/japplphysiol.00063.2011

Kozlovskaia, I. B. (2008). Fundamental and applied objectives of investigations in immersion [in Russian]. Aviakosm. Ekolog. Med. 42, 3-7.

Leach Huntoon, C. S., Grigoriev, A. I., and Natochin, Y. U.V. (1998). "Fluid and Electrolyte Regulation in Spaceflight. Science and Technology Series 94," in Am Astronaut. Society, San Diego.

Liang, X., Zhang, L., Wan, Y., Yu, X., Guo, Y., Chen, X., et al. (2012). Changes in the diurnal rhythms during a 45-day head-down bed rest. PLoS ONE 7:e47984. doi: 10.1371/journal.pone.0047984

Mano, T. (2005). Autonomic neural functions in space. Curr. Pharm. Biotechnol. 6, 319-324. doi: 10.2174/1389201054553743

Millet, C., Custaud, M. A., Maillet, A., Allevard, A. M., Duvareille, M., GauquelinKoch, G., et al. (2001). Endocrine responses to 7 days of head-down bed rest and orthostatic tests in men and women. Clin. Physiol. 21, 172-183. doi: 10.1046/j.1365-2281.2001.00315.x

Miwa, C., Sugiyama, Y., Iwase, S., Mano, T., Ohira, Y., Grigoriev, A., et al. (1997). Effects of three days of dry immersion on heart rate and blood pressure variabilities during head-up tilting in humans. Environ. Med. 41, 135-137.

Navasiolava, N. M., Custaud, M. A., Tomilovskaya, E. S., Larina, I. M., Mano, T., Gauquelin-Koch, G., et al. (2011a). Long-term dry immersion: review and prospects. Eur. J. Appl. Physiol. 111, 1235-1260. doi: 10.1007/s00421-010-1750-x

Navasiolava, N. M., de Germain, V., Levrard, T., Larina, I. M., Kozlovskaya, I. B., Diquet, B., et al. (2011b). Skin vascular resistance in the standing position increases significantly after 7 days of dry immersion. Auton. Neurosci. 160, 64-68. doi: 10.1016/j.autneu.2010.10.003

Navasiolava, N. M., Pajot, A., Gallois, Y., Pastushkova, L., Kulchitsky, V. A., Gauquelin-Koch, G., et al. (2011c). NT-ProBNP levels, water and sodium homeostasis in healthy men: effects of 7 days of dry immersion. Eur. J. Appl. Physiol. 111, 2229-2237. doi: 10.1007/s00421-011-1858-7

Nicogossian, A. E., Huntoon, C. L., and Pool, S. L. (1993). Space Physiology and Medicine, $3 r d$ Edn. Philadelphia, PA: Lea and Febiger.

Panferova, N. E. (1976). Cardiovascular system during hypokinesia of varying duration and intensity [in Russian, English summary]. Kosm. Biol. Aviakosm. Med. 10, 15-20.

Pavy-Le Traon, A., Heer, M., Narici, M. V., Rittweger, J., and Vernikos, J. (2007). From space to earth: advances in human physiology from 20 years of bed rest studies (1986-2006). Eur. J. Appl. Physiol. 101, 143-194. doi: 10.1007/s00421-007-0474-z

Pavy-Le Traon, A., Louisy, F., Vasseur-Clausen, P., Güell, A., and Gharib, C. (1999). Contributory factors to orthostatic intolerance after simulated weightlessness. Clin. Physiol. 19, 360-368. doi: 10.1046/j.1365-2281.1999.00189.x

Protheroe, C. L., Ravensbergen, H. R., Inskip, J. A., and Claydon, V. E. (2013). Tilt testing with combined lower body negative pressure: a "gold standard" for measuring orthostatic tolerance. J. Vis. Exp. 73:e4315. doi: 10.3791/4315

Schmidt, W., and Prommer, N. (2005). The optimised CO-rebreathing method: a new tool to determine total haemoglobin mass routinely. Eur. J. Appl. Physiol. 95, 486-495. doi: 10.1007/s00421-005-0050-3

Shulzhenko, E. B., Tigranyan, R. A., Panfilov, V. E., and Bzhalava, I. I. (1980). Physiological reactions during acute adaptation to reduced gravity. Life Sci. Space Res. 18, 175-179. doi: 10.1016/B978-0-08-024436-5.50023-8

Treffel, L., Dmitrieva, L., Gauquelin-Koch, G., Custaud, M. A., Blanc, S., Gharib, C., et al. (2016a). Craniomandibular system and postural balance after 3day dry immersion. PLoS ONE 11:e0150052. doi: 10.1371/journal.pone.0 150052

Treffel, L., Mkhitaryan, K., Gellee, S., Gauquelin-Koch, G., Gharib, C., Blanc, S., et al. (2016b). Intervertebral disc swelling demonstrated by $3 \mathrm{D}$ and water content magnetic resonance analyses after a 3-day dry immersion simulating microgravity. Front. Physiol. 7:605. doi: 10.3389/fphys.2016.00605 
Vernikos, J., Ludwig, D. A., Ertl, A. C., Wade, C. E., Keil, L., and O’Hara, D. (1996). Effect of standing or walking on physiological changes induced by head down bed rest: implications for spaceflight. Aviat. Space Environ. Med. 67, 1069-1079.

Watenpaugh, D. E. (2016). Analogs of microgravity: head-down tilt and water immersion. J. Appl. Physiol. 120, 904-914. doi: 10.1152/japplphysiol.00986.2015

Yuan, M., Alameddine, A., Coupé, M., Navasiolava, N. M., Li, Y., GauquelinKoch, G., et al. (2015). Effect of Chinese herbal medicine on vascular functions during 60-day head-down bed rest. Eur. J. Appl. Physiol. 115, 1975-1983. doi: 10.1007/s00421-015-3176-y

Zhang, L. F. (2001). Vascular adaptation to microgravity: what have we learned? J. Appl. Physiol. 91, 2415-2430.
Conflict of Interest Statement: The authors declare that the research was conducted in the absence of any commercial or financial relationships that could be construed as a potential conflict of interest.

Copyright $\odot 2017$ De Abreu, Amirova, Murphy, Wallace, Twomey, GauquelinKoch, Raverot, Larcher, Custaud and Navasiolava. This is an open-access article distributed under the terms of the Creative Commons Attribution License (CC BY).

The use, distribution or reproduction in other forums is permitted, provided the original author(s) or licensor are credited and that the original publication in this journal is cited, in accordance with accepted academic practice. No use, distribution or reproduction is permitted which does not comply with these terms. 